\title{
Homocysteine as a risk factor for nephropathy and retinopathy in Type 2 diabetes
}

\author{
H. C. Looker ${ }^{1}$, A. Fagot-Campagna ${ }^{3}$, E. W. Gunter ${ }^{3}$, C. M. Pfeiffer ${ }^{3}$, K. M. Venkat Narayan ${ }^{3}$, W. C. Knowler ${ }^{2}$, \\ R. L. Hanson ${ }^{2}$ \\ 11550 E Indian School Road, Phoenix, USA \\ ${ }^{2}$ Diabetes and Arthritis Epidemiology section, NIDDK, Phoenix, Arizona, USA \\ ${ }^{3}$ Center for Disease Control, Atlanta, Georgia, USA
}

\section{Abstract}

Aims/hypothesis. The aim of this study was to examine the relation between serum total homocysteine concentrations and microvascular complications in Pima Indians with Type 2 diabetes.

Methods. Homocysteine concentrations were measured in frozen sera of 396 diabetic participants in a longitudinal study who were 40 years of age or older and who had attended one or more examinations between 1982 and1985. Retinopathy was assessed by fundoscopy and nephropathy by an albumin:creatinine ratio greater than $300 \mathrm{mg} / \mathrm{g}$. The incidence rate ratio for a $5 \mu \mathrm{mol} / \mathrm{l}$ difference in homocysteine was calculated using proportional hazard regression.

Results. The incidence of each complication was assessed in subjects without that complication at baseline and with more than one follow-up examination: 229 for nephropathy, 212 for retinopathy and 266 for proliferative retinopathy. There were 101 incident cases of nephropathy, 113 of retinopathy and 40 of proliferative retinopathy during a mean follow-up of 8.6, 7.5 and 8.9 years, respectively. Incidence of neph- ropathy was associated with homocysteine concentrations: IRR $=1.42(95 \% \mathrm{CI}, 1.09-1.84, p=0.01)$; this remained statistically significant controlled for age, sex and duration of diabetes $(p=0.03)$, but not when controlled for baseline renal function $(p=0.4)$. Homocysteine concentrations were not associated with the incidence of any retinopathy IRR $=1.14(95 \% \mathrm{CI}$ $0.89-1.46, p=0.3$ ) but were associated with the incidence of proliferative retinopathy $\mathrm{IRR}=1.62(95 \% \mathrm{CI}$ $1.16-2.28, p=0.005)$; this association remained statistically significant when controlled for baseline renal function and diabetes duration ( $p=0.02)$.

Conclusions/interpretation. Increased homocysteine concentrations are associated with an increased risk for incidence of nephropathy and proliferative retinopathy; the relation with incidence of nephropathy seems to be explained by an association with baseline albuminuria status concentrations, whereas the relation with incidence of proliferative retinopathy does not. [Diabetologia (2003) 46:766-772]

Keywords Homocysteine, diabetic retinopathy, diabetic nephropathy, diabetes mellitus, type 2 .
An association between severely increased serum homocysteine concentrations $(>100 \mu \mathrm{mol} / \mathrm{l})$, as seen in homocystinuria, and the early onset of arteriosclerotic

Received: 11 November 2002 / Revised: 24 January 2003

Published online: 28 May 2003

C) Springer-Verlag 2003

Corresponding author: Dr. H. C. Looker, 1550 E Indian School Road, Phoenix, Arizona 85014, USA

E-mail: hlooker@mail.nih.gov

Abbreviations: IRR, Incident rate ratio; ACR, albumin to creatinine ratio. disease has long been recognized, although the mechanism that links homocysteine to vascular disease is not yet clear. This has prompted studies to see if more modestly increased concentrations of homocysteine $(15-100 \mu \mathrm{mol} / \mathrm{l})$ are associated with arteriosclerotic disease $[1,2]$. As macrovascular disease is a common complication of diabetes, studies have also examined the role of homocysteine and the incidence of cardiovascular disease in diabetes [3]. In addition associations have been reported between homocysteine concentrations in diabetic subjects and the prevalence of microvascular disease, such as nephropathy $[4,5]$ and 
retinopathy $[6,7]$. An association between baseline homocysteine and the incidence of microalbuminuria in a largely non-diabetic population has also been reported [8]. We investigated the relation of homocysteine and the prevalence and incidence of microvascular complications in Pima Indians with Type 2 diabetes.

\section{Subjects and methods}

The present study was part of an ongoing longitudinal study amongst the Pima Indians of the Gila River Indian Community in central Arizona. Community residents are invited to undergo examinations on a biennial basis regardless of health. These examinations include anthropometric measurements, direct fundoscopy through dilated pupils, retinal photography, urinalysis, systolic and diastolic blood pressure and fasting, and 2-h plasma glucose taken as part of a $75 \mathrm{~g}$ OGTT. Use of medication, current alcohol intake (classified into none, light or moderate to heavy) and smoking history (classified as either current smoker or not) were also recorded during each exam. Albuminuria was assessed by nephelometry (Hyland Nephelometer 4/82-12/83, Baker Nephelometer 1/84-5/88, Behring Nephelometer $6 / 88$ to present). Blood pressure was measured to the nearest $2 \mathrm{mmHg}$ with a mercury sphygmomanometer with diastolic blood pressure measured at the $4^{\text {th }}$ Korotkoff sound. Hypertension was defined as a systolic blood pressure above $140 \mathrm{mmHg}$, a diastolic blood pressure above $90 \mathrm{mmHg}$ or use of antihypertensive medication. Diabetes was diagnosed on the basis of a $75 \mathrm{~g}$ glucose tolerance test according to the World Health Organization guidelines or of a documented previous clinical diagnosis.

We selected all diabetic participants in the longitudinal study who had an examination during the years 1982 to 1985 and who were 40 years of age or older at that time. Stored sera were available for 396 people ( $80 \%$ of the 493 people otherwise eligible). Total serum homocysteine was measured by HPLC with fluorometric detection [9] in 1998 on fasting serum samples, when available, which had been stored at $-20^{\circ} \mathrm{C}$. For 21 subjects we did not have fasting samples available and 2-h sera were used instead. Exclusion of the data from the 2-h samples did not change the overall results of the analyses. Vitamin $\mathrm{B}_{12}$ and folate were also measured using a competitive protein binding radioimmunoassay from Bio-Rad Laboratories (Hercules, Calif., USA) [10]. Vitamin $\mathrm{B}_{12}$ and folate concentrations were available for 394 of the 396 subjects.

Fundoscopy and urinary albumin measurements were available for 371 subjects at the baseline visit and 289 subjects had at least one follow-up exam by December 31st, 2000 in which fundoscopy and/or urinary albumin measurements were available (73\% of all those with available homocysteine concentrations). Among those without nephropathy at baseline $(n=283)$ $229(81 \%)$ had at least one follow-up examination and likewise among those without retinopathy at baseline $(n=271) 212$ (78\%) had at least one follow-up examination; of those without proliferative retinopathy at baseline $(n=349) 266(76 \%)$ had at least one follow up examination. For a subgroup of 266 subjects, baseline retinal photographs were also available with 178 subjects also having a follow-up retinal photograph.

We assessed the association of baseline homocysteine with the presence and development of nephropathy and retinopathy. Nephropathy was defined by a urine albumin to creatinine ratio (ACR) of $300 \mathrm{mg} / \mathrm{g}$ or more. Retinopathy was classified into three groups: none, non-proliferative, and proliferative. Grad- ing was based on the fundoscopic findings of the worst eye at each exam. The "none" group included eyes with hard exudates only, as previously they have been shown to be common in Pima Indians without diabetes [11]. The "non-proliferative" group comprised those with at least a single microaneurysm in one eye but no signs of proliferative retinopathy. The "proliferative" group included those with proliferative changes or scars from previous laser therapy in at least one eye.

Baseline data were analysed using Spearman rank correlation coefficients for the continuous variables whereas linear regression models were used to compare geometric means of homocysteine concentrations for the categorical variables (sex, hypertension, smoking, use of antidiabetic medicine). Linear regression models were also used to calculate age and sex adjusted geometric means for homocysteine, vitamin $B_{12}$ and folate at baseline for the presence or absence of each complication. The natural logarithm was taken for each of the dependent variables to reduce skewness. One subject had severe hyperhomocysteinemia (of $103 \mu \mathrm{mol} / \mathrm{l}$ as well as nephropathy and proliferative retinopathy) at baseline. This individual was excluded from the analyses because the primary aim of this study was to evaluate the role of more modest concentrations of serum homocysteine and such extreme values could be unduly influential in statistical models. In incidence analyses person-time accumulated from the baseline exam to the first exam at which a complication was present or until the most recent exam attended (as of the end of 2000) if the complication remained absent, allowing a maximal follow-up of 18 years. Homocysteine was included in univariate and multivariate analyses for the development of nephropathy or retinopathy using Cox's proportional hazard models. Various models included potentially confounding variables such as age, sex, duration of diabetes, fasting plasma glucose concentrations, urine ACR, serum creatinine and hypertension. Fasting glucose was used as a measure of glycaemia in preference to $\mathrm{HbA}_{1}$ as there were less missing data, though results were similar when $\mathrm{HbA}_{1}$ was used in the models instead. Subjects were included in the models when they did not have the relevant complication (nephropathy, any retinopathy or proliferative retinopathy) at baseline. The validity of the proportionality assumption was tested for each variable by including a time-dependent interaction term [12]. Incidence rate ratios (IRRs) with 95\% CI are expressed for a $5 \mu \mathrm{mol} / \mathrm{l}$ difference in serum total homocysteine which is approximately equal to one standard deviation. A $p$ value of less than 0.05 was considered statistically significant.

All participants in this study gave their informed consent and this research was carried in accordance with the Declaration of Helsinki as revised in 2000. The longitudinal Pima study is approved by the institutional review board of the National Institute of Diabetes and Digestive and Kidney Diseases and by the Gila River Indian Community. The homocysteine study was also approved by the institutional review board of the Centers for Disease Control and Prevention.

\section{Results}

Baseline demographics and laboratory data are shown along with baseline correlations between homocysteine concentrations and other continuous variables (Table 1). The correlation coefficient was the highest for serum creatinine and homocysteine concentrations $(r=0.51, p<0.0001)$. Age, ACR, systolic and diastolic blood pressure were also positively correlated with homocysteine concentrations while BMI, fasting glu- 
Table 1. Baseline measures and spearman correlation coefficients with serum homocysteine concentrations for study participants

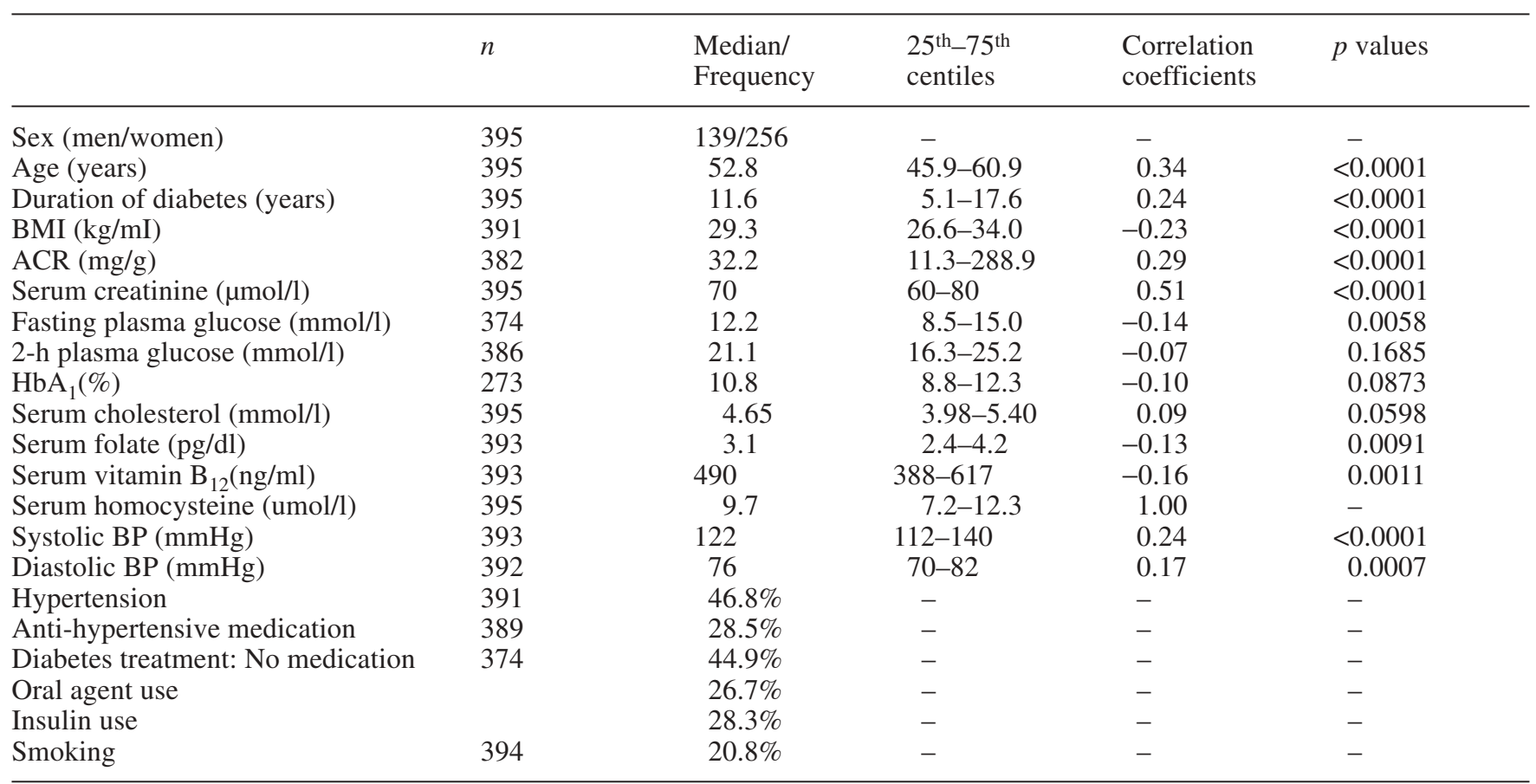

$p$ values are given for the null hypothesis that the correlation coefficient is 0

Table 2. Homocysteine and the prevalence of microvascular complications

\begin{tabular}{llcccc}
\hline & & $\mathrm{N}$ & Homocysteine $^{\mathrm{a}}(\mu \mathrm{mol} / \mathrm{l})$ & Vitamin B12 $(\mathrm{ng} / \mathrm{ml})_{\mathrm{Folate}(\mathrm{pg} / \mathrm{ml})}$ \\
\hline \multirow{2}{*}{ Nephropathy } & None & $289^{\mathrm{c}}$ & $8.99(8.62-9.38)$ & $490(468-515)$ & $3.25(3.05-3.46)$ \\
& Present & 93 & $10.88(10.09-11.73)^{\mathrm{b}}$ & $478(439-522)$ & $3.22(2.88-3.59)$ \\
Retinopathy & None & 279 & $9.23(8.84-9.65)$ & $498(476-522)$ & $3.24(3.04-3.46)$ \\
& Non-proliferative & 79 & $9.71(8.94-10.59)$ & $451(414-493)$ & $3.08(2.72-3.48)$ \\
& Proliferative & $23^{\mathrm{d}}$ & $9.89(8.49-11.53)$ & $526(447-619)$ & $3.33(2.64-4.19)$ \\
\hline
\end{tabular}

a Geometric means adjusted for age and sex (95\% CI)

b Statistically significantly different from no nephropathy group $(p<0.0001)$

cose, vitamin $\mathrm{B}_{12}$ and folate were negatively correlated with homocysteine concentrations. In linear regression models men had a higher concentration of homocysteine than the women $(10.6 \mu \mathrm{mol} / \mathrm{l}$ vs $8.9 \mu \mathrm{mol} / \mathrm{l}$ respectively, $p<0.0001)$. Hypertensive subjects had higher concentrations than normotensive subjects $(10.7 \mu \mathrm{mol} / 1$ vs $8.6 \mu \mathrm{mol} / 1$ respectively, $p<0.0001)$ but there was no statistically significant difference between homocysteine concentrations in hypertensive subjects treated with anti-hypertensive medication and those who were untreated $(p=0.8)$. People who did not undergo pharmacologic treatment for diabetes had a lower mean concentration of homocysteine than those who were taking insulin (either alone or in combination with oral hypoglycaemic agents) $(9.6 \mu \mathrm{mol} / \mathrm{l}$ vs $9.9 \mu \mathrm{mol} / \mathrm{l}$ respectively, $p=0.60$ ) but had a higher mean concentration than those who were taking only oral hypoglycaemic agents $(9.6 \mu \mathrm{mol} / 1$ vs $8.7 \mathrm{umol} / \mathrm{l}$ respectively, $p=0.0513)$. There was no statistically sig- c 287 with vitamin B12 and folate measures

d 22 with vitamin B12 and folate measures

nificant association with smoking $(9.4 \mu \mathrm{mol} / \mathrm{l}$ for non smokers and $9.6 \mu \mathrm{mol}$ for current smokers, $p=0.72$ ) or alcohol consumption $(9.4 \mu \mathrm{mol} / \mathrm{l}$ for non drinkers vs $9.6 \mu \mathrm{mol} / \mathrm{l}$ for light alcohol drinkers and $8.7 \mu \mathrm{mol} / \mathrm{l}$ for moderate to heavy drinkers, $p=0.64)$. In a linear regression model including all factors with significant correlation $(p<0.05)$ to homocysteine at baseline, serum creatinine, serum folate (inverse), age and male sex were independently associated with homocysteine concentrations.

Baseline homocysteine was higher in those with than without nephropathy but there was no association with baseline retinopathy grades (Table 2). Folate and Vitamin $\mathrm{B}_{12}$ were not associated with either nephropathy or retinopathy at baseline (Table 2).

Development of Nephropathy. Of the 229 people without nephropathy at baseline, 101 developed nephropathy over a mean follow-up period of 8.6 years (Inci- 
Table 3. Incident rate ratios for nephropathy $(\mathrm{ACR} \geq 300 \mathrm{mg} / \mathrm{g})$ per $5 \mu \mathrm{mol} / \mathrm{l}$ difference in baseline serum homocysteine concentration

IRR $95 \% \mathrm{CI}$

Development of nephropathy_all participants (229 subjects and 101 events)

Univariate

Adjusted for vitamin B12 and folate

Adjusted for age and sex

Adjusted for age, sex and diabetes duration

Adjusted for age, sex, diabetes duration and systolic BP

Adjusted for age, sex, diabetes duration and fasting glucose

Adjusted for age, sex, diabetes duration and ACR

Adjusted for age, sex diabetes duration and serum creatinine $1.42^{\mathrm{a}}$

$1.43^{\mathrm{a}}$

1.33

$1.39^{\mathrm{a}}$

$1.38^{\mathrm{a}}$

1.21

1.12

1.19
$1.09-1.84$

$1.10-1.87$

$1.00-1.77$

$1.04-1.86$

$1.03-1.84$

$0.92-1.58$

$0.87-1.45$

$0.86-1.66$

Development of nephropathy — participants with normoalbuminuria at baseline (ACR <30 mg/g) (152 subjects and 48 events)

Univariate

Adjusted for vitamin B12 and folate

Adjusted for age and sex

Adjusted for age, sex and diabetes duration

Adjusted for age, sex, diabetes duration and systolic BP

Adjusted for age, sex, diabetes duration and fasting glucose

Adjusted for age, sex, diabetes duration and ACR

Adjusted for age, sex, diabetes duration and serum creatinine $1.60^{\mathrm{a}}$

$1.59^{\mathrm{a}}$

$1.68^{\mathrm{a}}$

$1.96^{\mathrm{a}}$

1.83

$1.90^{\mathrm{a}}$

1.63

$1.77^{\mathrm{a}}$
$1.01-2.54$

$1.00-2.53$

$1.04-2.71$

$1.17-3.30$

$0.99-3.37$

$1.12-3.21$

$0.95-2.79$

$1.06-2.95$

IRR, Incidence rate ratio; ${ }^{\mathrm{a}} p<0.05$

dence Rate $=51.2 / 1000$ person years). Homocysteine concentrations were associated with the incidence of nephropathy and remained so when adjusted for age, sex, duration of diabetes and systolic blood pressure (Table 3). Results were similar when hypertension was included as a categorical variable. However, the IRR was markedly attenuated (and was no longer statistically significant) when baseline fasting glucose concentrations, ACR or creatinine were included in the model. Although the analysis was limited to subjects without nephropathy at baseline, both baseline ACR and serum creatinine concentrations were positively associated with the incidence of nephropathy in univariate models. (ACR had an IRR of $2.70,95 \%$ CI 2.14-3.42 per $10 \mathrm{mg} / \mathrm{g}$ difference, and serum creatinine had an IRR of $3.08,95 \%$ CI $0.84-11.22$ per $10 \mu \mathrm{mol} / \mathrm{l}$ difference).

We repeated our analyses restricted to those who did not have microalbuminuria at baseline $(\mathrm{ACR}<30 \mathrm{mg} / \mathrm{g})$. In these analyses there were 152 people with 48 incident cases over a follow-up period of 9.8 years (Incidence Rate $=32.1 / 1000$ person years). The point estimates for incident rate ratios were stronger in all these models than in those for the full set of 229 people without baseline nephropathy and were not substantially attenuated by adjustment for confounders, although the association was not statistically significant when adjusted for systolic blood pressure or ACR.

Development of any retinopathy. There were 212 people with "no retinopathy" on fundoscopy at baseline. Over a mean follow-up of 7.5 years, 113 people developed retinopathy (non-proliferative or proliferative
Incidence Rate $=70.8 / 1000$ person years, Table 4). Homocysteine was not a statistically significant risk factor for the development of any retinopathy in either univariate or multivariate analyses (Table 4). This was still the case when the baseline group was restricted to people with normoalbuminuria at baseline. We found similar results for the subgroup with retinal photograph data for whom retinopathy could be defined.

Development of proliferative retinopathy. Of the 266 people without proliferative retinopathy at baseline, 40 developed proliferative retinopathy over a mean follow-up period of 8.9 years (Incidence Rate $=17.0 / 1000$ person years). Homocysteine had an incident rate ratio of 1.62 (95\% CI $1.16-2.28)$ per $5 \mu \mathrm{mol} / \mathrm{l}$ difference for the development of proliferative retinopathy as diagnosed with fundoscopy in univariate analysis and this was only slightly attenuated in models adjusted for age, sex and duration of diabetes (Table 4). Due to the small number of incident cases we were limited in the number of variables that could be included without overfitting the model and, as age and sex were not in themselves statistically significant factors they were not included in all the models shown. Further adjustment for ACR, serum creatinine or fasting glucose concentrations only modestly attenuated the IRR associated with homocysteine though the result for the model with serum creatinine, diabetes duration and fasting glucose was not quite statistically significant. Using a forward stepwise selection procedure to which all variables other than homocysteine were available, we identified insulin use and serum creatinine as the only statistically significant predictors of proliferative retinopathy. The risk associated with homocysteine 
Table 4. Incident rate ratios for retinopathy per $5 \mu \mathrm{mol} / \mathrm{l}$ difference in baseline serum homocysteine concentration

IRR

Development of any retinopathy (212 subjects and 113 events)

Univariate

Adjusted for vitamin B12 and folate

Adjusted for age and sex

Adjusted for age, sex and diabetes duration

Adjusted for age, sex, diabetes duration and ACR

Adjusted for age, sex, diabetes duration and serum creatinine

Adjusted for age, sex, diabetes duration and systolic BP

Development of proliferative retinopathy (266 subjects and 40 events)

Univariate

Adjusted for vitamin B12 and folate

Adjusted for age and sex

Adjusted for age, sex and diabetes duration

Adjusted for diabetes duration and ACR

Adjusted for diabetes duration and serum creatinine

Adjusted for diabetes duration, ACR and systolic BP

Adjusted for diabetes duration, ACR and fasting glucose

Adjusted for diabetes duration, serum creatinine and fasting glucose

Adjusted for insulin use and serum creatinine
1.14

1.14

1.05

1.03

1.01

0.94

1.04

$1.62^{\mathrm{a}}$

$1.61^{\mathrm{a}}$

$1.58^{\mathrm{a}}$

$1.54^{\mathrm{a}}$

$1.53^{\mathrm{a}}$

1.41

$1.47^{\mathrm{a}}$

$1.49^{\mathrm{a}}$

1.36

1.44
(95\% CI)

0.89-1.46

0.89-1.46

$0.80-1.37$

$0.79-1.36$

$0.61-1.42$

0.63-1.39

$0.79-1.37$

1.16-2.28

1.14-2.27

1.08-2.32

$1.05-2.28$

$1.07-2.20$

0.95-2.10

1.02-2.13

1.04-2.12

0.92-2.02

$0.97-2.13$

IRR, incidence rate ratio; ${ }^{\mathrm{a}} p<0.05$

was only modestly attenuated by adjustment for these two variables, IRR=1.44 (95\% CI 0.97-2.13, Table 4).

Restricting the group to those with normoalbuminuria at baseline $(A C R<30 \mathrm{mg} / \mathrm{g})$ reduced the number of incident cases to 16 but the univariate analysis still showed homocysteine to be a statistically significant risk factor (IRR=2.08, 95\%CI 1.20-2.62) though the number of cases was too small for further models.

Vitamin B12 and Folate. Both vitamin B12 and folate were negatively correlated with homocysteine but neither was associated with the incidence of microvascular complications. Including these variables in models with homocysteine made little change to the IRR estimates for any complication (Table 3, Table 4).

\section{Discussion}

Among Pima Indians with diabetes, serum homocysteine concentrations were higher in the presence of nephropathy and the incidence of nephropathy was associated with baseline homocysteine concentrations. There was no association between homocysteine concentrations and the prevalence or incidence of retinopathy but higher serum homocysteine was associated with increased risk for proliferative retinopathy.

High concentrations of homocysteine have been associated with macrovascular disease and cardiovascular mortality in both the general population [1] and in people with diabetes [3]. In many populations homocysteine concentrations are higher in smokers [13] but that was not the case in our study; this finding could relate to the lower prevalence of smoking in this population [14].

It has been reported that homocysteine concentrations are increased in renal failure of various aetiologies [15] though the mechanism for this remains unclear. Several studies have found higher mean homocysteine concentrations associated with prevalence of nephropathy in people with Type 2 diabetes $[4,5,16]$. Similar findings have also been reported for Type 1 diabetes with correlations to albumin excretion rate $[4,6,17]$ and serum creatinine [17] which are similar to our results in Pima Indians with Type 2 diabetes.

There is little information on whether homocysteine concentrations predict future incidence of nephropathy. In a population study from the Netherlands, higher baseline homocysteine concentrations have been associated with an increased incidence of microalbuminuria. This association was statistically significant in people without diabetes but the number of subjects with diabetes was too small for meaningful conclusions [8]. In our study, increased homocysteine concentrations at baseline were associated with an increased incidence of subsequent nephropathy, but this association was much attenuated with control for baseline ACR or serum creatinine concentrations. This suggests that increased homocysteine concentrations per se do not lead to renal damage, but they could serve as a marker of occult nephropathy. If so, the finding that the risk was stronger and was not attenuated with control for baseline ACR or serum creatinine in those with normoalbuminuria suggests that homocysteine could be a particularly sensitive 
marker of renal function in these subjects. We have defined nephropathy on the basis of increased urinary albumin excretion. Although we can not completely exclude nephropathy due to non-diabetic causes, in this population increased albumin excretion in the presence of diabetes is virtually always associated with evidence of diabetic nephropathy on biopsy [18, 19].

There have been a number of studies concerning the relation between homocysteine and retinopathy. A high prevalence of retinopathy has been found amongst people with Type 2 diabetes who had fasting homocysteine concentrations greater than $16 \mu \mathrm{mol} / \mathrm{l}$ [20]. This study reported an odds ratio adjusted for age, sex, $\mathrm{HbA}_{1 \mathrm{c}}$, hypertension and creatinine clearance of 3.33 (95\% CI 0.99-11.19). Other studies in Type 1 diabetes have reported positive associations between retinopathy and homocysteine $[4,6,7,17]$. In some studies this relation seems to be confounded by nephropathy $[4,17]$, whereas in others it persists despite control for nephropathy $[6,7]$. In our study, there was no association between homocysteine concentrations and prevalence of retinopathy in Pima Indians with Type 2 diabetes.

There are few data on the relation between homocysteine and the incidence of diabetic retinopathy. In a longitudinal study of 50 people with Type 1 diabetes followed for 5 years, baseline homocysteine concentrations were correlated with serum creatinine and urinary albumin excretion but not with baseline or incident retinopathy [21]. We also showed no relation between homocysteine concentrations and incidence of retinopathy of any grade but there was an association between increased homocysteine and incidence of proliferative retinopathy. The strength of this relation was only modestly attenuated with control for baseline albuminuria or glycaemia. Although the association between homocysteine and risk of proliferative retinopathy could be explained by homocysteine being a marker of renal function or other risk factors for proliferative retinopathy, homocysteine seems to be a sensitive marker that provides additional information about risk of proliferative retinopathy beyond that provided by customary clinical measures.

We made use of homocysteine concentrations measured in stored serum samples. The possibility of homocysteine degradation during long-term storage has been examined by a study which measured homocysteine concentrations in sera frozen for 6 to 16 years at $-20^{\circ} \mathrm{C}$, and compared them with fresh samples. The difference in homocysteine concentrations was not correlated to storage time over that time frame [22]. We also compared homocysteine from stored samples with fresh samples in 20 subjects and found that while homocysteine was slightly higher in the older samples this difference was not statistically significant for fasting samples. It is customary that EDTA whole blood collected for homocysteine measurements is either separated at once from the red cells or stored over ice for up to $4 \mathrm{~h}$ after drawing the blood to avoid an artificial increase of homocysteine concentrations through the red cells that are still producing and releasing homocysteine into the plasma. That was not the case with our fasting samples, which could have been held up to $2 \mathrm{~h}$ before processing. We did find that homocysteine concentrations were slightly higher in the fasting samples than in the freshly collected and optimally processed samples for the 20 subjects (which were mostly processed within an hour of drawing), but these differences were again not statistically significant. Furthermore it is unlikely that any artificial increase or degradation influenced the results since all the fasting samples were processed identically. Since initial drawing the majority of samples included in this study (354/396) had been thawed and refrozen on one occasion before homocysteine was measured. We have no data on how this could affect measured concentrations.

Our study has relied on the use of a single baseline measure homocysteine concentration and other covariates for predicting outcomes. Whereas this is analogous to the clinical situation, it could be informative for biologic purposes to consider exposure over the entire period at risk (i.e. in a time-dependent analysis). We could not look at homocysteine as a time-dependent variable as repeat measures were not made. One would expect incidence rate ratios based on a single measure to be closer to the null hypothesis than those based on time-dependent covariates so we do not think that this undermines the positive associations we found with nephropathy and proliferative retinopathy. We were unable to assess dietary folate intake as that was not recorded in our study but we were able to show that the association was not likely to be mediated by folate because serum folate was not a statistically significant risk factor. Although we have collected data on whether antihypertensive medications were being used we do not have data on which types of antihypertensives were being taken. Thus it is difficult to determine whether specific medications for hypertension could have been a confounding factor in this study. However, others have found no difference in homocysteine concentrations between differing hypertensive medications including diuretics and ACE inhibitors [23].

It is possible that increased homocysteine is more than a marker of disease and is actually a causative factor in the development of nephropathy and proliferative retinopathy. In which case control for baseline creatinine could be overadjusting. If so, the mechanism by which this occurs could be similar to that by which it presumably causes macrovascular disease. The mechanism by which homocysteine is related to macrovascular disease is not clear [24]. Homocysteine has been shown to damage endothelial cells via the generation of hydrogen peroxide [25] and to alter 
vasomotor activity [26]. In vitro work has suggested that this damage could be potentiated in the presence of hyperglycaemia [27]. There is also evidence that homocysteine can cause apoptosis in retinal ganglion cells of mice [28]. Whether homocysteine is a marker for microvascular disease risk or actually has a causal role for people with diabetes, homocysteine could become a useful measurement for assessing both macrovascular and microvascular risk in diabetes.

Acknowledgements. We wish to thank the members of the Gila River Indian Community and everyone at the NIH clinic in Sacaton, Arizona. We also thank Prof. K. Aleck for his advice during the preparation of this manuscript.

\section{References}

1. Giles WH, Croft JB, Greenlund KJ, Ford ES, Kittner SJ (2000) Association between total homocyst(e)ine and the likelihood for a history of acute myocardial infarction by race and ethnicity: Results from the Third National Health and Nutrition Examination Survey. Am Heart J 139:446453

2. Eikelboom JW, Lonn E, Genest J Jr, Hankey G, Yusuf S (1999) Homocyst(e)ine and cardiovascular disease: a critical review of the epidemiologic evidence. Ann Intern Med 1999 131:363-375

3. Hoogeveen EK, Kostense PJ, Beks PJ et al. (1998) Hyperhomocysteinemia is associated with an increased risk of cardiovascular disease, especially in non-insulin-dependent diabetes mellitus: a population-based study. Arterioscler Thromb Vasc Biol 18:133-138

4. Chico A, Perez A, Cordoba A et al. (1998)Plasma homocysteine is related to albumin excretion rate in patients with diabetes mellitus: a new link between diabetic nephropathy and cardiovascular disease? Diabetologia 41:684-693

5. Buysschaert M, Dramais AS, Wallemacq PE, Hermans MP (2000) Hyperhomocysteinemia in type 2 diabetes: relationship to macroangiopathy, nephropathy, and insulin resistance. Diabetes Care 23:1816-1822

6. Chiarelli F, Pomilio M, Mohn A et al. (2000) Homocysteine levels during fasting and after methionine loading in adolescents with diabetic retinopathy and nephropathy. J Pediatr 137:386-392

7. Vaccaro O, Perna AF, Mancini FP et al. (2000) Plasma homocysteine and microvascular complications in type 1 diabetes. Nutr Metab Cardiovasc Dis 10:297-304

8. Jager A, Kostense PJ, Nijpels G et al. (2001)Serum homocysteine levels are associated with the development of (micro)albuminuria: the Hoorn study. Arterioscler Thromb Vasc Biol 21:74-81

9. Pfeiffer CM, Huff DL, Gunter EW (1999) Rapid and accurate HPLC assay for plasma total homocysteine and cysteine in a clinical laboratory setting. Clin Chem 45:290 292

10. Gunter EW, Lewis BL, Koncikowski SM (1998) Laboratory methods used for the Third National Health and Nutrition Examination Survey (NHANES III), 1988-1994. Centers for Disease Control and Prevention, Hyattsville, MD
11. Knowler WC, Bennett PH, Ballintine EJ (1980) Increased incidence of retinopathy in diabetics with elevated blood pressure. A six-year follow-up study in Pima Indians. N Engl J Med 302:645-650

12. Prentice RL, Kalbfleisch JD (1979) Hazard rate models with covariates. Biometrics 35:25-39

13. Jacques PF, Bostom AG, Wilson PW et al. (2001) Determinants of plasma total homocysteine concentration in the Framingham Offspring cohort. Am J Clin Nutr 73:613-621

14. Welty TK, Lee ET, Yeh J et al. (1995) Cardiovascular disease risk factors among American Indians. The Strong Heart Study. Am J Epidemiol 142:269-287

15. Chauveau P, Chadefaux B, Coude M et al. (1993) Hyperhomocysteinemia, a risk factor for atherosclerosis in chronic uremic patients. Kidney Int Suppl 41:S72-S77

16. Emoto M, Kanda H, Shoji T et al. (2001) Impact of insulin resistance and nephropathy on homocysteine in type 2 diabetes. Diabetes Care 24:533-538

17. Agardh CD, Agardh E, Andersson A, Hultberg B (1994) Lack of association between plasma homocysteine levels and microangiopathy in type 1 diabetes mellitus. Scand J Clin Lab Invest 54:637-641

18. Pagtalunan ME, Miller PL, Jumping-Eagle S et al. (1997) Podocyte loss and progressive glomerular injury in type II diabetes. J Clin Invest 99:342-348

19. Kamenetzky SA, Bennett PH, Dippe SE, Miller M, LeCompte PM (1974) A clinical and histologic study of diabetic nephropathy in the Pima Indians. Diabetes 23:6168

20. Hoogeveen EK, Kostense PJ, Eysink PE et al. (2000) Hyperhomocysteinemia is associated with the presence of retinopathy in type 2 diabetes mellitus: the Hoorn study. Arch Intern Med 160:2984-2990

21. Hultberg B, Agardh CD, Agardh E, Lovestam-Adrian M (1997) Poor metabolic control, early age at onset, and marginal folate deficiency are associated with increasing levels of plasma homocysteine in insulin-dependent diabetes mellitus. A five-year follow-up study. Scand J Clin Lab Invest 57:595-600

22. Israelsson B, Brattstrom L, Refsum H (1993) Homocysteine in frozen plasma samples. A short cut to establish hyperhomocysteinaemia as a risk factor for arteriosclerosis? Scand J Clin Lab Invest 53:465-469

23. Sharabi Y, Doolman R, Rosenthal T et al. (1999) Homocysteine levels in hypertensive patients with a history of cardiac or cerebral atherothrombotic events. Am J Hypertens 12:766-771

24. Welch GN, Loscalzo J (1998) Homocysteine and atherothrombosis. N Engl J Med 338:1042-1050

25. Starkebaum G, Harlan JM (1986) Endothelial cell injury due to copper-catalyzed hydrogen peroxide generation from homocysteine. J Clin Invest 77:1370-1376

26. Chen C, Conklin BS, Ren Z, Zhong DS (2002) Homocysteine decreases endothelium-dependent vasorelaxation in porcine arteries. J Surg Res 102:22-30

27. Hofmann MA, Kohl B, Zumbach MS et al. (1998) Hyperhomocyst(e)inemia and endothelial dysfunction in IDDM. Diabetes Care 21:841-848

28. Moore P, El Sherbeny A, Roon P et al. (2001)Apoptotic cell death in the mouse retinal ganglion cell layer is induced in vivo by the excitatory amino acid homocysteine. Exp Eye Res 73:45-57 